\title{
Effect of Polymorphisms of Three Genes Mediating Monoamine Signalling on Brain Morphometry in Schizophrenia and Healthy Subjects
}

\author{
Anupa A. Vijayakumari ${ }^{1,2}$, John P. John ${ }^{1,2,4}$, Harsha N. Halahalli ${ }^{3, *}$, Pradip Paul ${ }^{2}$, Priyadarshini Thirunavukkarasu ${ }^{1,2}$, \\ Meera Purushottam², Sanjeev Jain ${ }^{2}$ \\ ${ }^{1}$ Multimodal Brain Image Analysis Laboratory (MBIAL), Departments of ${ }^{2}$ Psychiatry, ${ }^{3}$ Neurophysiology, and ${ }^{4}$ Clinical Neuroscience, National \\ Institute of Mental Health and Neurosciences (NIMHANS), Bangalore, India
}

\begin{abstract}
Objective: We examined the effect of risk alleles of polymorphisms of three schizophrenia risk genes that mediate monoamine signalling in the brain on regional brain volumes of schizophrenia and healthy control subjects. The risk alleles and the gene polymorphisms studied were: Val allele of catechol o-methyltransferase (COMT) rs4680 polymorphism; short allele of 5-hydroxy tryptamine transporter linked polymorphic region (5HTTLPR) polymorphism; and T allele of 5-hydroxy tryptamine 2A (5HT2A) rs6314 polymorphism.

Methods: The study was carried out on patients with recent onset schizophrenia $(n=41)$ recruited from the outpatient department of National Institute of Mental Health and Neurosciences, Bangalore, India and healthy control subjects ( $n=39$ ), belonging to South Indian Dravidian ethnicity. Individual and additive effects of risk alleles of the above gene polymorphisms on brain morphometry were explored using voxel-based morphometry.

Results: Irrespective of phenotypes, individuals with the risk allele T of the rs6314 polymorphism of 5HT2A gene showed greater (at cluster-extent equivalent to family wise error-correction [FWEc] $p<0.05$ ) regional brain volumes in the left inferior temporal and left inferior occipital gyri. Those with the risk alleles of the other two polymorphisms showed a trend (at $p<0.001$, uncorrected) towards lower regional brain volumes. A trend (at $p<0.001$, uncorrected) towards additive effects of the above 3 risk alleles (subjects with 2 or 3 risk alleles vs. those with 1 or no risk alleles) on brain morphology was also noted.

Conclusion: The findings of the present study have implications in understanding the role of individual and additive effects of genetic variants in mediating regional brain morphometry in health and disease.
\end{abstract}

KEY WORDS: Magnetic resonance imaging; Gene polymorphism; Catechol-O-methyl transferase; 5-Hydroxy tryptamine 2A; 5-Hydroxy tryptamine transporter linked polymorphic region; Voxel-based morphometry.

\section{INTRODUCTION}

Brain morphometric abnormalities have been extensively reported in schizophrenia since the advent of modern neuroimaging techniques in the mid-1970s. It is intriguing to note that, apart from reduced whole brain volume, ${ }^{1)}$ there are no brain regions that have been consistently shown to exhibit morphometric abnormalities

Received: June 9, 2014 / Revised: August 18, 2014

Accepted: October 19, 2014

Address for correspondence: John P. John, MD

Department of Psychiatry, National Institute of Mental Health and

Neurosciences (NIMHANS), PB No 2900 Dharmaram P.O., Hosur

Road, Bangalore Karnataka 560 029, India

Tel: +091-80-26995329/26995250, Fax: +091-80-26564492/ 26564822

E-mail: jpjnimhans@gmail.com

${ }^{*}$ Current affiliation of Harsha N. Halahalli is Department of

Physiology, K.S. Hegde Medical Academy, Nitte University,

Mangalore, Karnataka, India across these various studies. ${ }^{2,3)}$ Differences in sample characteristics such as age, gender distribution, duration of illness, exposure to neuroleptics, drug use etc. could contribute towards the variability of morphometric findings across studies. ${ }^{4-8)}$ However, a substantial number of studies, especially over the past decade or so have attempted to control for these confounding variables at the sample selection and/or analysis phases. ${ }^{910)}$ Despite this, no consistent morphometric abnormalities that are associated with the schizophrenia phenotype have emerged from these studies. This begs the question, whether there could be other factors that could be different across the various samples in morphometric studies of schizophrenia. The obvious candidate would be the variability of the constitution of genotypic risk variants that impact brain volumes across study samples, given our understanding that schizophrenia is a polygenic disorder. ${ }^{11)}$

(a) This is an Open-Access article distributed under the terms of the Creative Commons Attribution Non-Commercial License (http://creativecommons.org/licenses/by-nc/3.0) which permits unrestricted non-commercial use, distribution, and reproduction in any medium, provided the original work is properly cited. 
These multiple genes of small effect ${ }^{12,13)}$ may have differential influence on regional brain volumes, thus contributing to the heterogeneity of the brain morphometric findings in schizophrenia. Moreover, gene-gene epistatic interactions as well as epigenetic alterations of gene expression may play a role in mediating regional brain volumes.

Genes that mediate monoamine signalling have been strongly implicated in schizophrenia. ${ }^{14,15}$ Catechol O-methyltransferase (COMT) is an enzyme involved in the degradation of catecholamine neurotransmitters like dopamine, epinephrine and norepinephrine. ${ }^{16)}$ In the exon 4 of COMT gene, G-to-A transition results in a valine (Val)-to-methionine (Met) substitution at amino acid position 108 or 158 leading to a four-fold decrease in enzyme activity in Met homozygotes. ${ }^{17)}$ Genome wide association studies and family based association studies have shown COMT as a risk gene for the development of schizophrenia. ${ }^{18-23)}$ There is considerable evidence for the association Val allele of COMT (rs4680) gene with schizophrenia. ${ }^{18,20)}$ Subjects with Val/Val genotype performed less well in tasks assessing working memory compared to Met/Met individuals, supporting the fact that the higher activity Val allele lowers the prefrontal dopamine concentration leading to cognitive deficits in schizophrenia. ${ }^{24,25)}$ Imaging studies have also linked the Val allele of Val145Met polymorphism with reduced gray matter volumes in various cortical and sub-cortical brain regions in schizophrenia. ${ }^{26,27)}$ The 5-hydroxy tryptamine $2 \mathrm{~A}$ (5HT2A) gene that mediates serotoninergic neurotransmission in the brain is another possible gene involved in the pathogenesis of schizophrenia. The nonsynonymous His452Tyr polymorphism is located in the exon 3 of 5HT2A receptor gene, where a C-T transition results in a histidine (His)-to-tyrosine (Tyr) substitution at the amino acid position $452 .{ }^{28)}$ In a recent study in Han Chinese population, it was shown that carriers of Tyr allele had greater risk for schizophrenia than subjects with CC genotype, suggesting that the Tyr allele might act as a susceptibility risk variant in the pathophysiology of schizophrenia. ${ }^{29)}$ In two independent studies, the frequency of Tyr allele was found to be higher in patients with schizophrenia. ${ }^{30,31)}$ Moreover, a large multi-centric study found an association between schizophrenia and another polymorphism in the coding region of the 5HT2A receptor gene, viz., the T102C polymorphism. ${ }^{32)}$ Gray matter volume reductions were noted in the temporal lobe region of healthy subjects who were carriers of Tyr allele of His452Tyr polymorphism. ${ }^{33)}$ Another candidate gene for schizophrenia that mediates monoamine signaling in the brain is the serotonin transporter gene (5HTT) which plays a major role in regulating the intensity and duration of serotonergic signalling at synapses. ${ }^{34)}$ Many studies have supported the role of the polymorphism 5HTT linked polymorphic region (5HTTLPR) as a risk factor for various symptom dimensions of schizophrenia. ${ }^{35-37)}$ The 5HTTLPR is located in the promoter region of the serotonin receptor. It is rendered polymorphic by a $44 \mathrm{bp}$ insertion/deletion resulting in a short (S) allele having lower transcription efficacy in comparison to the long (L) allele. ${ }^{38)}$ In South Indian population, it was shown that patients with schizophrenia had higher homozygous genotypic frequencies of the short allele of 5HTTLPR. ${ }^{39)}$ Carriers of the short allele of 5HTTLPR have shown reduced gray matter volume in perigenual anterior cingulate cortex and amygdala in healthy subjects. ${ }^{40)}$ However, a post mortem study has reported that subjects with schizophrenia who were homozygous for the short allele of 5HTTLPR gene polymorphism had a higher number and volume of pulvinar neurons in the thalamus. ${ }^{41)}$

Previous studies that have reported phenotypic differences in regional brain volumes in schizophrenia have ignored genotypic variability across the study samples. Therefore, in the present study, we planned to group subjects with schizophrenia and healthy subjects depending on the presence or absence of risk alleles of schizophrenia risk gene polymorphisms that affect monoamine signaling, ignoring the phenotype. Using an imaging-genomics approach, we examined whether there were significant regional volumetric abnormalities associated with polymorphisms of the above three schizophrenia risk genes that mediate monoamine signalling in the brain, viz., COMT, 5HTT and 5HT2A. Based on the findings of previous genetic association, imaging and neuropsychological studies reviewed above, we considered Val (G) allele of COMT rs4680 polymorphism, ${ }^{11,42)}$ short (S) allele of 5HTTLPR polymorphism ${ }^{39)}$ and $\mathrm{T}$ allele of 5HT2A rs6314 polymorphism ${ }^{30,31)}$ as the risk alleles for studying the effect of polymorphisms of the above risk genes on brain morphometry. A voxel-based morphometric (VBM) approach was employed to carry out morphometric comparisons between carriers and non-carriers of the risk alleles on each of these three risk gene polymorphisms, irrespective of phenotype. An additive model was also employed to investigate the cumulative effect of risk alleles of these polymorphisms on regional gray matter volumes. We hypothesized that the risk alleles of the above gene polymorphisms would exert differential effects, indivi- 
dually as well as additively, on regional brain volumes.

\section{METHODS}

\section{Study Samples}

The study was carried out at the National Institute of Mental Health and Neurosciences (NIMHANS), Bangalore, India, with due approval from the NIMHANS Ethics Committee thus conforming to the ethical standards laid down in the 1964 Declaration of Helsinki. Written informed consent was obtained after detailed explanation of the study protocol from all the subjects (and their accompanying relatives in the case of all individuals with schizophrenia) prior to enrolment into the study. The above informed consent process was reviewed by the NIMHANS Ethics Committee prior to providing the approval for initiating the study at NIMHANS.

The study samples comprised of 41 patients with recent-onset schizophrenia and 39 healthy control subjects (HCS). The diagnosis of schizophrenia or schizophreniform disorder was arrived at using criteria from the Diagnostic and Statistical Manual for Mental Disorders fourth edition (DSM-IV) based on the consensus of a research psychiatrist who conducted a semi-structured interview and a trained research assistant who used the Mini International Neuropsychiatric Interview (MINI) Plus.
Only those patients who did not meet criteria for any other Axis I disorder, including substance dependence (other than nicotine) as per MINI-Plus, with an age of first onset of psychotic symptoms at or after 17 years of age and a duration of illness less than or equal to 5 years were recruited into the study. All patients had predominant positive symptoms and had diagnoses of paranoid $(n=30)$, undifferentiated $(\mathrm{n}=8)$ and schizophreniform $(\mathrm{n}=7)$ subtypes. The patients with schizophreniform disorder included in the morphometric analysis comprised of only those who were re-diagnosed as schizophrenia during follow-up and who retained a diagnosis of schizophrenia even after 1-3 years, as inferred from follow-up data obtained from case files. The baseline severity of schizophrenia psychopathology was evaluated using the Positive and Negative Syndrome Scale (PANSS) ${ }^{43)}$ by two trained raters who had established good inter-rater reliability. The history of exposure to antipsychotics was ascertained by interviewing the patient and relative/s, and corroborated from available medical records. Twenty five of the forty one patients with schizophrenia were not on neuroleptics, of which 17 were drug naïve at the time of recruitment into the study. The remaining patients were on antipsychotics, the cumulative doses of which were converted to 'risperidone equivalents, ${ }^{44-46)}$ HCS were ascertained to be free from Axis I or II psychiatric disorders using the MINI-Plus.

Table 1. Socio-demographic and clinical characteristics of the study samples

\begin{tabular}{|c|c|c|}
\hline \multirow{2}{*}{ Characteristic } & \multicolumn{2}{|c|}{ Participant $(n=80)$} \\
\hline & Healthy control group $(n=39)$ & Schizophrenia group $(n=41)$ \\
\hline \multicolumn{3}{|l|}{ Gender } \\
\hline Male & 32 & 29 \\
\hline Female & 7 & 12 \\
\hline Age $(y r)$ & $26.26 \pm 5.85$ & $27.34 \pm 7.48$ \\
\hline Education (formal education, yr) & $13.41 \pm 3.603$ & $12.61 \pm 3.13$ \\
\hline \multicolumn{3}{|l|}{ Diagnosis } \\
\hline Paranoid schizophrenia & & 27 \\
\hline Undifferentiated schizophrenia & & 8 \\
\hline Schizophreniform disorder & & 6 \\
\hline \multicolumn{3}{|l|}{ PANSS score } \\
\hline Positive & & $14.42 \pm 6.77$ \\
\hline Negative & & $14.27 \pm 6.43$ \\
\hline Global & & $25.97 \pm 8.94$ \\
\hline Total & & $54.67 \pm 17.32$ \\
\hline Age at onset of illness (yr) & & $25.29 \pm 7.65$ \\
\hline Duration of illness (mo) & & $24.54 \pm 17.97$ \\
\hline \multicolumn{3}{|l|}{ Medication status* } \\
\hline Antipsychotic naive/free & & $17 / 8$ \\
\hline On medication & & 16 \\
\hline $\begin{array}{l}\text { Life-time cumulative neuroleptic exposure in } \\
\text { risperidone equivalent dosages (mg) }\end{array}$ & & $1,352.54 \pm 1,961.54$ \\
\hline
\end{tabular}

Values are presented as number only or mean \pm standard deviation.

*Life-time cumulative neuroleptic exposure expressed in risperidone equivalents (mg). ${ }^{31-33)}$

PANSS, Positive and Negative Syndrome Scale 
Current use/abuse of psychotropic drugs as well as history of psychiatric illness in first-degree relatives in the HCS was ruled out by an unstructured clinical interview. Both the schizophrenia and healthy control groups comprised only of subjects hailing from the southern part of India belonging to Dravidian ethnicity. The demographic and clinical characteristics of the samples are given in Table 1.

\section{Magnetic Resonance Imaging (MRI)}

MRI scans were performed on a Philips Achieva 3.0 T scanner using a SENSE-8 head coil (Philips, Andover, MA, USA). Head movements were minimized by applying a band over the forehead during the scanning procedure. A high-resolution T1-weighted MRI volume data set of the whole brain with a resolution of $1 \times 1 \times 1 \mathrm{~mm}^{3}$ (field of view: $256 \times 256 \mathrm{~mm}$; acquisition matrix: $256 \times 256$ ) was acquired using an Magnetization Prepared Rapid Gradient Echo (MPRAGE) sequence: repetition time $=8.2 \mathrm{~ms}$, echo time $=3.8 \mathrm{~ms}$, flip angle $=8^{\circ}$, sense factor: 3.5 . All scans were inspected for artifacts and gross structural abnormalities by an expert neuroradiologist.

\section{Image Analysis}

Image processing and analysis was done using the VBM8 toolbox (Christian Gaser's VBM8 toolbox; http:// dbm.neuro.uni-jena.de/) within Statistical Parametric Mapping version 8 software (www.fil.ion.ucl.ac.uk/spm) running under Matlab R2012a. The T1 weighted structural images were bias-corrected and segmented into gray matter (GM), white matter (WM) and cerebrospinal fluid (CSF) maps based on a set of Tissue Probability Maps provided in the VBM8 toolbox. ${ }^{47)}$ The GM maps were normalized using the standard MNI152 DARTEL (Diffeomorphic Anatomical Registration through Exponentiated Lie algebra) template. Finally, GM maps were spatially smoothed with a Gaussian kernel of $8 \mathrm{~mm}$ full-widthat-half-maximum. The total GM, WM generated from the VBM analysis were added to generate the total brain volume (TBV).

\section{Genotyping}

Venous blood sample of approximately $10 \mathrm{ml}$ was collected from all the study participants. The DNA was extracted from the blood samples by modified salting out method. ${ }^{48)}$ Polymerase chain reaction (PCR) using tetra-primer amplification refractory mutation system was used to amplify the Val158Met (rs4680) polymorphism of COMT gene. The following forward primer 5'-CCAACC CTGCACAGGCAAGAT-3', reverse primer 5'-CAAGG
GTGACCTGGAACAGCG-3', forward primer 5'-CGG ATGGTGGATTTCGCTGaCG-3', and reverse primer 5'TCAGGCATGCACACCTTGTCCTTtAT- $3{ }^{, 49)}$ was used for amplification. The gel products were visualized in $1.5 \%$ gel. Products expected were of $626 \mathrm{bp}$ and $222 \mathrm{bp}$ in Met158 homozygotes, $626 \mathrm{bp}$ and $451 \mathrm{bp}$ in Val158 (G allele) homozygotes and $626 \mathrm{bp}, 222 \mathrm{bp}$ and $451 \mathrm{bp}$ in the heterozygotes.

The His452Tyr (rs6314) polymorphism of 5HT2A gene was amplified by PCR-restriction fragment length polymorphism method using forward primer 5'-CAAAG CAAGATGCCAAG ACA-3' and reverse primer 5'-GGC ATACAGATATGATCGTTGG-3, ${ }^{28)}$ PCR products were subjected to restriction digestion at $37^{\circ} \mathrm{C}$ for 3 hours. The presence of $\mathrm{C}$ (Tyr) allele creates a restriction site for the restriction enzyme BbvI (National England Biolabs) with a product size of 180 and $68 \mathrm{bp}$. The T allele product size was $248 \mathrm{bp}$. The digested products were visualized in $2 \%$ gel after electrophoretic separation.

The $44 \mathrm{bp}$ insertion/deletion polymorphism in the promoter region of 5HTT gene, viz., the 5-HTTLPR polymorphism, was amplified by PCR. The following forward primer 5'-TCCTCCGCTTTGGCGCCTCTTCC-3' and reverse primer 5'-TGGGGGTTGCAGGGGAGATCCTG3 ' was used for the detection of 5HTTLPR. The amplified short (469 bp) and long (512 bp) allele products were visualized in $4 \%$ gel after electrophoretic separation. ${ }^{50)}$

\section{Statistical Analysis}

The genotype distribution of COMT (rs4680), 5HTT (5HTTLPR) and 5HT2A (rs6314) polymorphisms was calculated and found to be in Hardy-Weinberg's equilibrium. Using the VBM toolbox, analysis of covariance (ANCOVA) within the framework of general linear model (GLM) was used to initially compare regional brain volumes between schizophrenia and HCS. Age, gender and TBV were entered as nuisance co-variates. Subsequently, regional brain volumes were compared between genotypes of COMT (rs4680), 5HTT (5HTTLPR) and 5HT2A (rs6314) polymorphisms, irrespective of phenotype. In previous morphometric studies of the effect of COMT Val158Met polymorphism on brain volumes, a dominant model (Val/Val and Val/Met carriers vs. Met/Met homozygotes) was shown to be associated with brain morphometric changes in healthy young adults. ${ }^{27,51)}$ Furthermore, a gene-dose effect of the Val allele in reducing gray matter density of anterior cingulate was demonstrated in subjects at high risk for psychosis. ${ }^{42}$ Therefore, we assumed a dominant model for the COMT Val158Met poly- 
morphism (Val/Val + Val/Met vs. Met/Met) to examine the effect of the risk allele, viz., Val on brain volumes. For the 5HTTLPR polymorphism too, a dominant model (SS and LS vs. LL) was assumed, since the short allele (S) was found to act in a dominant fashion. ${ }^{52)}$ There were no subjects homozygous for the risk $\mathrm{T}$ allele in the case of 5HT2A (rs6314) polymorphism and therefore group comparisons were made between TC and CC genotypes. Thus, the overall sample $(\mathrm{N}=80$ [schizophrenia $+\mathrm{HCS}$ ]) was sub-divided according to the COMT (rs4680), 5HTT (5HTTLPR) and 5HT2A (rs6314) genotypes, irrespective of phenotype viz., COMT: Val/Val + Val/Met vs. Met/Met (i.e., GG + GA vs. AA); 5HTTLPR: $S / S+L / S$ vs. $L / L$ (i.e., $\mathrm{SS}+\mathrm{LS}$ vs. LL); and 5HT2A: tyrosine-cysteine (TC) vs. cysteine-cysteine (CC). Using a full factorial model in VBM, we examined the interaction between diagnosis and each of the candidate genotypes (COMT [rs4680], 5HTT [5HTTLPR] and 5HT2A [rs6314]). We then carried out exploratory post-hoc comparisons of regional brain volumes between the genotype-wise sub-groups for each of the above 3 polymorphisms. Additive effect of the risk alleles on brain morphometry was explored by comparing brain volumes of subjects who had 2 or 3 risk alleles $(n=53)$ of the above 3 risk genes vs. those who had 1 or no risk alleles $(\mathrm{n}=27)$. In view of the limited number of subjects having the risk allele $\mathrm{T}(\mathrm{n}=13)$ we attempted to find the additive effect of the other 2 risk alleles (Val of COMT rs4680 and S allele of 5HTTLPR) after excluding the subjects who were carriers of the T allele of 5HT2A rs6314 polymorphism $(n=14)$ by performing VBM comparison between those subjects having Val allele of COMT and short allele of 5HTT $(n=40)$ vs. those subjects having 1 or no risk alleles $(n=26)$ of COMT rs4680 and 5HTTLPR.

Age, gender and TBV were entered as nuisance co-variates for all the above analyses. Given the exploratory na- ture of this study, we used 3 levels of statistical thresholds; initially at a stringent statistical significance threshold of family wise error (FWE) $p<0.05$ (no significant differences emerged at this stringent threshold); then, with cluster-extent correction equivalent to the cluster extent of FWE $p<0.05$, at a height threshold of $p<0.001$ uncorrected $^{53)}$; and, finally, uncorrected $(p<0.001)$ for multiple comparisons with a voxel extent threshold of $\mathrm{k}=20$ (trend-level findings). Generating the results of group-wise analyses using different thresholds for statistical significance in an exploratory study like this would provide information regarding the level of confidence that one may have about an individual finding.

\section{RESULTS}

The genotype and allelic frequency distribution of the COMT rs4680, 5HTTLPR and 5HT2A rs6314 polymorphisms in schizophrenia and healthy control samples are given in Table 2. There were no significant allele- or genotype-wise differences between the healthy and schizophrenia groups for all the three candidate genes.

VBM analysis between phenotypes (schizophrenia vs. HCS) did not show definitive volumetric differences at a significance threshold of FWE $p<0.05$. Gray matter volume reductions were noted in the left anterior culmen and posterior declive in patients with schizophrenia when compared to HCS (at cluster-extent equivalent to FWE correction [FWEc] $p<0.05$; cluster defining threshold: $p_{\text {uncorrected }}<0.001 ; \mathrm{k}=793$ voxels) (Fig. 1A, Table 3 ). At an uncorrected threshold $(p<0.001,20$ voxel extent threshold), patients with schizophrenia showed gray matter volume reductions in bilateral parahippocampal gyri, bilateral anterior culmen, left posterior declive, right middle temporal gyrus, left inferior and middle frontal gyri, left posterior tuber and right uncus (Fig. 1B, Table 4).

Table 2. Genotype and allele frequency distribution of schizophrenia and healthy control samples

\begin{tabular}{|c|c|c|c|c|c|c|c|c|c|}
\hline \multirow{2}{*}{ Gene } & \multirow{2}{*}{ SNP } & \multirow{2}{*}{ Alleles } & \multicolumn{2}{|c|}{ Allele frequency } & \multirow{2}{*}{ Genotypes } & \multicolumn{2}{|c|}{ Genotype frequency } & \multirow{2}{*}{$\begin{array}{l}\text { Pearson's } \\
\text { chi-square }\end{array}$} & \multirow{2}{*}{$\begin{array}{l}\text { Asymptotic } \\
\text { significance } \\
\text { (two-sided) }\end{array}$} \\
\hline & & & $S Z(n=41)$ & $\mathrm{HC}(n=39)$ & & $S Z(n=41)$ & $H C(n=39)$ & & \\
\hline \multirow[t]{3}{*}{ COMT } & rs4680 & $A$ & 0.37 & 0.52 & $A A$ & $8(0.19)$ & $11(0.28)$ & 3.897 & 0.143 \\
\hline & & $G$ & 0.63 & 0.48 & $A G$ & $15(0.37)$ & $19(0.49)$ & & \\
\hline & & & & & GG & $18(0.44)$ & $9(0.23)$ & & \\
\hline \multirow[t]{3}{*}{ 5HTR2A } & rs6314 & $\mathrm{C}$ & 0.92 & 0.89 & $\mathrm{CC}$ & $35(0.85)$ & $31(0.79)$ & 1.115 & 0.573 \\
\hline & & $T$ & 0.07 & 0.10 & TC & $6(0.15)$ & $8(0.21)$ & & \\
\hline & & & & & $\pi$ & - & - & & \\
\hline \multirow[t]{3}{*}{$5 \mathrm{HTT}$} & 5HTTLPR & L & 0.42 & 0.48 & LL & $8(0.19)$ & $9(0.23)$ & 0.489 & 0.564 \\
\hline & & $S$ & 0.58 & 0.52 & LS & $18(0.44)$ & $20(0.51)$ & & \\
\hline & & & & & SS & $15(0.37)$ & $10(0.26)$ & & \\
\hline
\end{tabular}

SZ, schizophrenia; HC, healthy controls. 
A

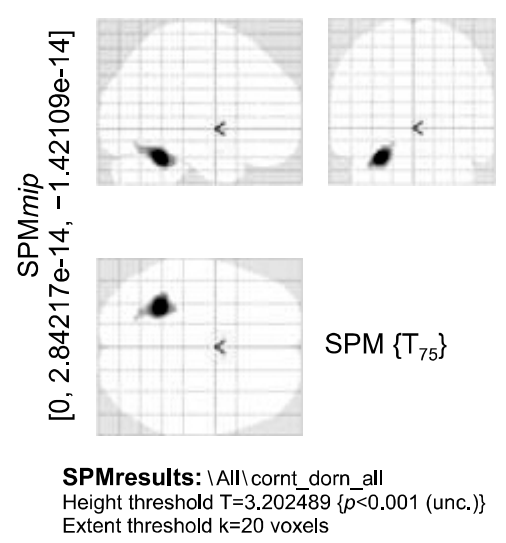

$\mathrm{SZ}<\mathrm{HCS}$
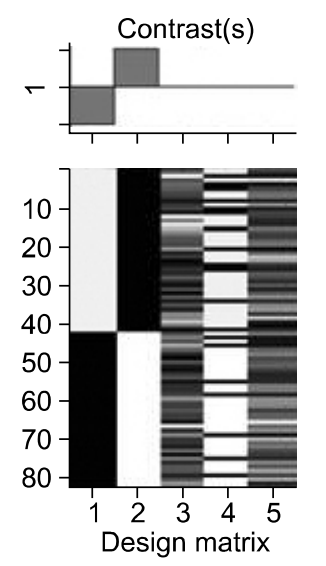

B

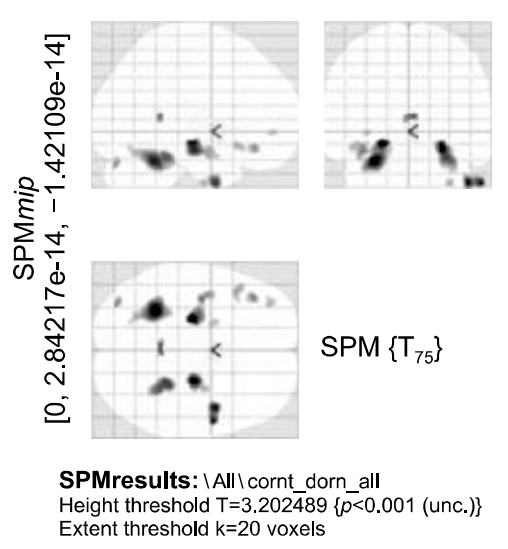

$\mathrm{SZ}<\mathrm{HCS}$

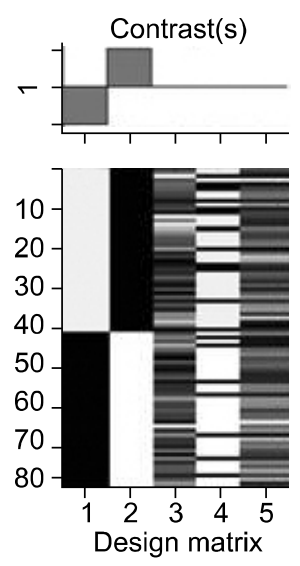

Fig. 1. Statistical parametric t-map showing reduced gray matter volumes in schizophrenia subjects (SZ, $\mathrm{n}=41$ ) in comparison to healthy subjects (HCS, $n=39$ ) at $(A)$ family wise error-correction-; height threshold: $p<0.001$; extent threshold=793 voxels, and (B) uncorrected significance threshold of $p<0.001$ and an extent threshold of 20 voxels. Total brain volume, age, and gender were entered in the two sample random effects analysis as co-variates. Display according to neurological convention (image left is participant's left). SPM, statistical parametric mapping.

Table 3. Brain regions showing reduced gray matter volumes in schizophrenia subjects $(n=41)$ in comparison to healthy subjects $(n=39)$

\begin{tabular}{lllll}
\hline$X$ coor & Y coor & Z coor & Brain region & Z score \\
\hline-27.23 & -45.65 & -22.13 & Left anterior culmen* & 3.955562 \\
-23.17 & -58.77 & -17.9 & Left posterior declive* & 3.271186 \\
\hline
\end{tabular}

FWEc corrected; height threshold $p<0.001$; extent threshold $=793$ voxels. Total brain volume, age and gender were entered in the two sample random effects analysis as co-variates.

*2 clusters significant at $p<0.001$ uncorrected; cluster with higher z-score listed.

FWEc, family wise error-correction.

VBM analysis using a full factorial model for examining the interaction between diagnosis and genotype did not yield significant results for COMT and 5HTTLPR gene polymorphisms. The 5HT2A polymorphism showed significant diagnosis $\mathrm{x}$ genotype interaction in left superior parietal lobule at FWE $(p<0.05)$ (Supplementary Table 1, Supplementary Fig. 1). However, post-hoc analyses of the effects of 5HT2A risk genotypes on brain morphometry separately in patients with schizophrenia and HCS did not yield any significant results at the corrected threshold.

Individuals with the risk allele $\mathrm{T}(\mathrm{n}=14)$ of 5 HT2A (rs6314) polymorphism had greater regional volumes in the left inferior temporal gyrus and left inferior occipital gyrus (FWEc; cluster defining threshold: $p_{\text {uncorrected }}$ $<0.001 ; \mathrm{k}=988$ voxels) in comparison to those homozygous for the $\mathrm{C}$ allele ( $\mathrm{n}=66$ ) (Fig. 2A, Table 5). At an uncorrected threshold ( $p<0.001,20$ voxel extent threshold), more brain regions bilaterally showed increased gray mat-
Table 4. Brain regions showing reduced gray matter volumes in schizophrenia subjects $(n=41)$ in comparison to healthy control subjects $(n=39)$

\begin{tabular}{crcll}
\hline X coor & Y coor & Z coor & \multicolumn{1}{c}{ Brain region } & Z score \\
\hline-20.37 & -17.52 & -7.19 & Left parahippocampal gyrus $^{\dagger}$ & 4.072562 \\
-27.23 & -45.65 & -22.13 & Left anterior culmen* $^{*}$ & 3.955562 \\
-19.05 & -64.65 & -15.68 & Left posterior declive* & 3.159514 \\
22.71 & -15.95 & -10.36 & Right parahippocampal gyrus & 3.896008 \\
41.1 & 2.94 & -31.23 & Right middle temporal gyrus & 3.772233 \\
25.53 & -36.28 & -19 & Right anterior culmen & 3.663617 \\
-35.47 & 30.46 & -6.95 & Left inferior frontal gyrus & 3.418089 \\
-32.82 & -74.71 & -27.68 & Left posterior tuber & 3.376054 \\
28.59 & -6.77 & -32.37 & Right uncus & 3.265416 \\
-32.8 & 41.98 & 5 & Left middle frontal gyrus & 3.213679 \\
\hline
\end{tabular}

Uncorrected significance threshold of $p<0.001$ and an extent threshold of 20 voxels. Total brain volume, age and gender were entered in the two sample random effects analysis as co-variates.

*2 clusters significant at $p<0.001$ uncorrected; cluster with higher z-score listed.

$z$-Score listed.
4 clusters significant at $p<0.001$ uncorrected; cluster with higher z-score listed.

ter volumes associated with the presence of the $\mathrm{T}$ allele (Fig. 2B, Table 6).

Genotype-wise comparisons of gray matter volumes using the dominant model for COMT and 5HTT polymorphisms revealed group differences only at an uncorrected threshold ( $p<0.001,20$ voxel extent threshold). Subjects with GG or AG genotypes of rs4680 of COMT $(n=61)$, irrespective of phenotype, showed decreased gray matter volumes in right anterior cingulate, bilateral medial frontal gyri, right declive, right superior temporal gyrus, right fusiform gyrus, right uncus, right frontal sub-gyral region and left lingual gyrus (Fig. 3, Table 7) compared to 


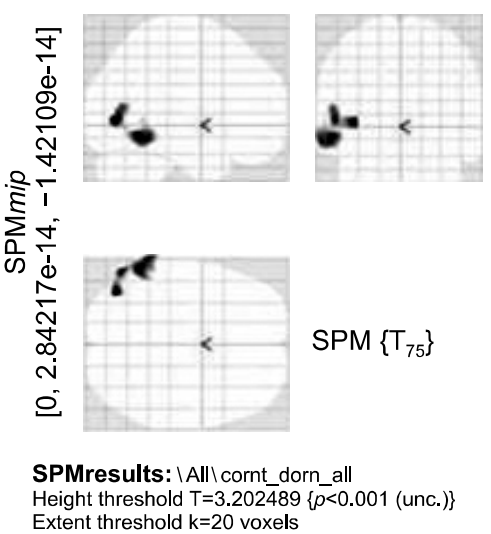

$\mathrm{TC}<\mathrm{CC}$

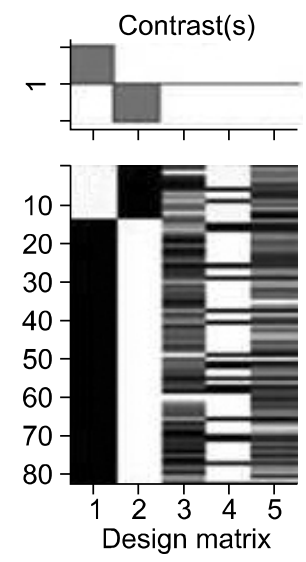

B

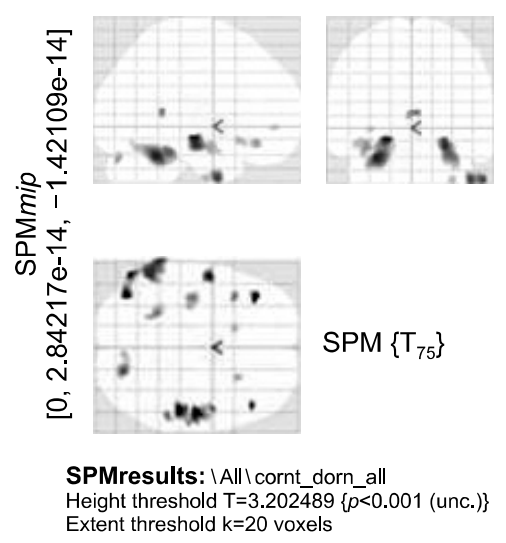

$\mathrm{TC}<\mathrm{CC}$

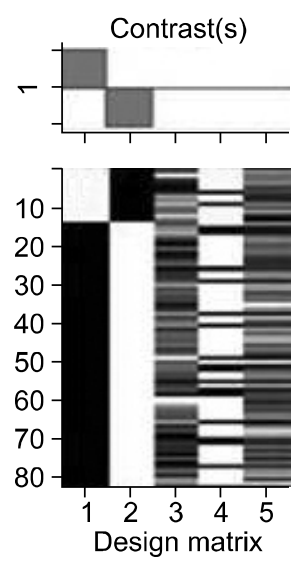

Fig. 2. Statistical parametric t-map showing increased gray matter volumes in subjects with the risk allele $\mathrm{T}$ of rs6314 (5HT2A) polymorphism $(n=14)$ in comparison to those homozygous for the $C$ allele $(n=66)$, irrespective of phenotype, at $(A)$ family wise error-correction threshold (height threshold: $p<0.001$; extent threshold=988 voxels) and (B) uncorrected significance threshold ( $p<0.001$ and an extent threshold of 20 voxels) irrespective of phenotype. Total brain volume, age, and gender were entered in the two sample random effects analysis as co-variates. Display according to neurological convention (image left is participant's left).

5HT2A, 5-hydroxy tryptamine 2A; TC, tyrosine- cysteine; CC, cysteine-cysteine; SPM, statistical parametric mapping.

Table 5. Brain regions showing showing increased gray matter volumes in subjects with the risk allele T of rs6314 (5HT2A) polymorphism $(n=14)$ in comparison to those homozygous for the C allele $(n=66)$ irrespective of phenotype

\begin{tabular}{rrrrr}
\hline$X$ coor & $Y$ coor & $Z$ coor & Brain region & $Z$ score \\
\hline-37.31 & -71.58 & -1.78 & Left inferior occipital gyrus & 4.024465 \\
-52.61 & -68.97 & 0.91 & Left inferior temporal gyrus* & 3.899168 \\
\hline
\end{tabular}

FWEc-corrected; height threshold: $p<0.001$; extent threshold=988 voxels. Total brain volume, age and gender were entered in the two sample random effects analysis as co-variates.

*2 clusters significant at $p<0.001$ uncorrected; cluster with higher z-score listed.

FWEC, family wise error-correction.

those individuals with AA genotype $(n=19)$. In case of 5HTTLPR, the individuals carrying SS or LS genotypes $(n=63)$ showed decreased gray matter volumes in the right anterior culmen and middle frontal gyrus when compared to L homozygotes ( $n=17$ ) (Fig. 4, Table 8).

Exploration of the additive effects of risk genes on brain volumes yielded differences between those subjects who had 2 or 3 risk alleles $(n=53)$ of the above 3 risk genes (high risk group) vs. those who had 1 or no risk alleles $(\mathrm{n}=27)$ (low risk group) at an uncorrected ( $p<0.001,20$ voxel extent threshold) threshold. The high risk group showed lower regional brain volumes in the right superior and right medial frontal gyri (Fig. 5, Table 9). When the exploration of additive effects was restricted to only those having the T allele of 5HT2A (rs6314) polymorphism along with one or two of the other two risk alleles $(n=13)$ vs. those who had one or no risk alleles $(n=27)$, the high risk group showed increased gray matter volumes in right
Table 6. Brain regions showing increased gray matter volumes in in subjects with the risk allele T of rs6314 (5HT2A) polymorphism $(n=14)$ in comparison to those homozygous for the C allele $(n=66)$ irrespective of phenotype

\begin{tabular}{cccll}
\hline X coor & Y coor & Z coor & \multicolumn{1}{c}{ Brain region } & Z score \\
\hline-34.27 & 29.02 & 7.8 & Left inferior frontal gyrus & 4.274454 \\
45.98 & -32.27 & 11.46 & Right superior temporal gyrus & 4.026 \\
54.29 & -24.19 & 15.07 & Right postcentral gyrus $^{\dagger}$ & 3.417343 \\
-37.31 & -71.58 & -1.78 & Left inferior occipital gyrus & 4.024465 \\
-52.61 & -68.97 & 0.91 & Left inferior temporal gyrus* & 3.899168 \\
43.45 & 25.82 & 8.81 & Right inferior frontal gyrus & 3.859638 \\
44.48 & -7.02 & 27.34 & Right precentral gyrus ${ }^{\dagger}$ & 3.668964 \\
17.3 & -71.13 & -37.3 & Right inferior semilunar lobule & 3.651302 \\
6.13 & -70.2 & -30 & Right uvula & 3.260589 \\
50.29 & -39.37 & -2.66 & Right middle temporal gyrus & 3.520164 \\
-35.69 & -20.5 & -5.02 & Left claustrum* & 3.493845 \\
-25.55 & -53.77 & 60.91 & Left superior parietal lobule & 3.480008 \\
-37.46 & 10.92 & 34.41 & Left precentral gyrus & 3.475083 \\
-55.76 & -52.53 & 34.84 & Left supramarginal gyrus & 3.431802 \\
-21.9 & -51.44 & -6.37 & Left parahippocampal gyrus & 3.35856 \\
22.8 & 17.58 & -7.18 & Right lentiform nucleus & 3.342297 \\
-11.93 & 13.44 & -6.81 & Left caudate & 3.246412 \\
\hline
\end{tabular}

Uncorrected significance threshold of $p<0.001$ and an extent threshold of 20 voxels. Total brain volume, age, and gender were entered in the two sample random effects analysis as co-variates. *2 clusters significant at $p<0.001$ uncorrected; cluster with higher z-score listed.

${ }^{+} 3$ clusters significant at $p<0.001$ uncorrected; cluster with higher z-score listed.

${ }^{\ddagger} 4$ clusters significant at $p<0.001$ uncorrected; cluster with higher z-score listed.

precentral gyrus, left inferior temporal gyrus, left inferior occipital gyrus, right inferior frontal gyrus and left superior parietal lobule (Supplementary Table 2, Supplementary Fig. 2). However, in view of the limited number of sub- 
$(G G+A G)<A A$

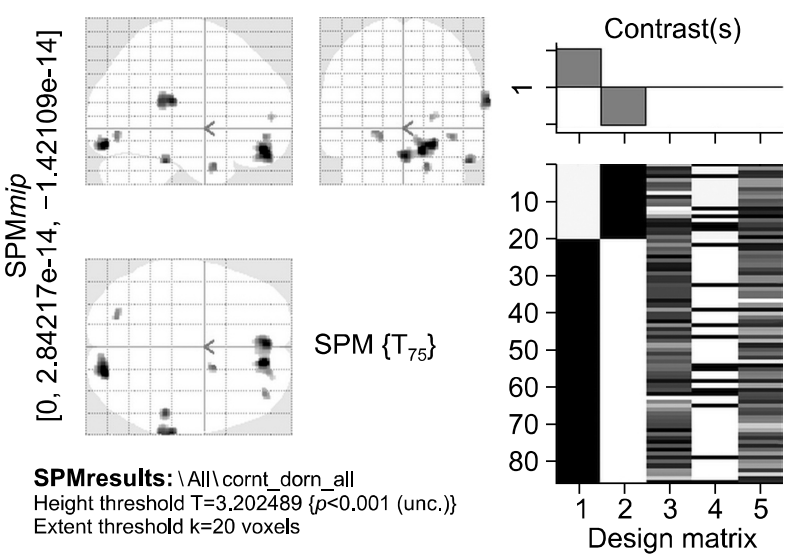

Fig. 3. Statistical parametric t-map showing reduced gray matter volumes associated with the $\mathrm{Val}$ allele of COMT rs4680 polymorphism, using dominant model ( Val/Val $(n=27)+$ Val/Met $(n=34)$ vs. Met/Met $(n=19)$ ), irrespective of phenotype, at uncorrected significance threshold of $p<0.001$ and an extent threshold of 20 voxels. Total brain volume, age, and gender were entered in the two sample random effects analysis as co-variates. Display according to neurological convention (image left is participant's left).

COMT, catechol o-methyltransferase; Val, valine; Met, methionine: GG, Val/Val; AG, Val/Met; SPM, statistical parametric mapping.

Table 7. Brain regions showing reduced gray matter volumes associated with the $\mathrm{Val}$ allele of COMT rs4680 polymorphism, using dominant model (Val/Val ( $n=27)+$ Val/Met ( $n=34)$ vs. Met/Met $(n=19)$ ) irrespective of phenotype

\begin{tabular}{crccc}
\hline X coor & $Y$ coor & Z coor & Brain region & Z score \\
\hline 11.8 & 43.06 & -7.66 & Right anterior cingulate & 4.053276 \\
-0.64 & 44.91 & -11.75 & Left medial frontal gyrus & 3.777444 \\
19.77 & -80.22 & -16.5 & Right cerebellum & 3.771318 \\
& & \multicolumn{4}{c}{ posterior declive } \\
61.16 & -31.61 & 18.53 & Right superior temporal gyrus* & 3.663792 \\
51.86 & -50.39 & -19.89 & Right fusiform gyrus* & 3.663373 \\
14.61 & 4.96 & -22.03 & Right uncus & 3.643609 \\
18.47 & 46.52 & 14.4 & Right medial frontal gyrus & 3.565357 \\
33.9 & 43.55 & 0.87 & Right sub-gyral & 3.404852 \\
-19.14 & -72.16 & -10.99 & Left lingual gyrus & 3.378122 \\
\hline
\end{tabular}

At uncorrected significance threshold of $p<0.001$ and an extent threshold of 20 voxels. Total brain volume, age, and gender were entered in the two sample random effects analysis as co-variates. *2 clusters significant at $p<0.001$ uncorrected; cluster with higher z-score listed.

jects having T risk allele of 5HTR2A ( $n=14)$, we repeated our analysis to explore additive effect of risk alleles by restricting our analysis to only those subjects without the $\mathrm{T}$ allele of 5HT2A $(\mathrm{N}=66)$. These subjects were grouped into those having Val allele of COMT and short allele of 5 HTT $(n=40)$ and those subjects having one or no risk alleles of the COMT and 5HTT polymorphisms $(n=26)$. The subjects with both risk alleles showed a trend towards re-
$(S S+L S)<L L$
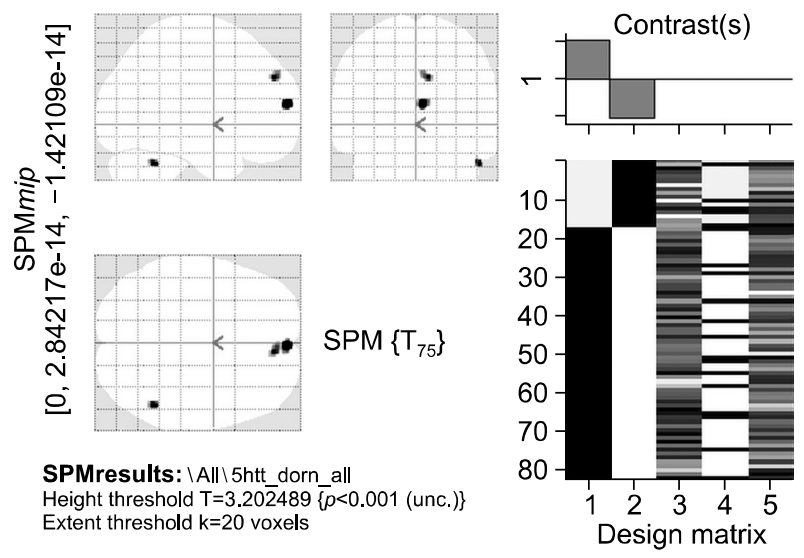

Fig. 4. Statistical parametric t-map map showing reduced gray matter volumes associated with the short allele of 5HTTLPR polymorphism, using the dominant model (short/short (SS, $\mathrm{n}=25$ ) and long/short ( $L S, n=38)+$ long/long $(L L, n=17)$ ), irrespective of phenotype, at an uncorrected significance threshold of $p<0.001$ and an extent threshold of 20 voxels. Total brain volume, age, and gender were entered in the two sample random effects analysis as co-variates. Display according to neurological convention (image left is participant's left).

5HTTLPR, 5-hydroxy tryptamine transporter linked polymorphic region; SPM, statistical parametric mapping.

Table 8. Brain regions showing reduced gray matter volumes associated with the short allele of 5HTTLPR polymorphism, using the dominant model (short/short $(n=25)$ and long/short $(n=38)+$ long/long $(n=17)$ ) irrespective of phenotype

\begin{tabular}{rrrcc}
\hline X coor & $Y$ coor & $Z$ coor & Brain region & $Z$ score \\
\hline 45.04 & -46.77 & -27.77 & Right anterior culmen & 3.480819 \\
7.03 & 40.03 & 37.92 & Right medial frontal gyrus & 3.436724 \\
\hline
\end{tabular}

At an uncorrected significance threshold of $p<0.001$ and an extent threshold of 20 voxels. Total brain volume, age, and gender were entered in the two sample random effects analysis as co-variates.

duction in gray matter volume in superior frontal, medial frontal, lingual, inferior parietal and anterior cingulate gyri in the right hemisphere and bilateral cerebellum at $p$ $<0.001 ; 20$ voxel extent threshold (Supplementary Table 3, Supplementary Fig. 3).

\section{DISCUSSION}

We examined the influence of polymorphisms of three schizophrenia candidate genes mediating monoamine signalling on regional brain volumes. We found greater regional brain volumes in left inferior temporal and left inferior occipital gyri in carriers of the $\mathrm{T}$ allele of rs6314 polymorphism of 5HT2A gene at a statistical significance threshold of FWEc $p<0.05$. Using a dominant model, Val 


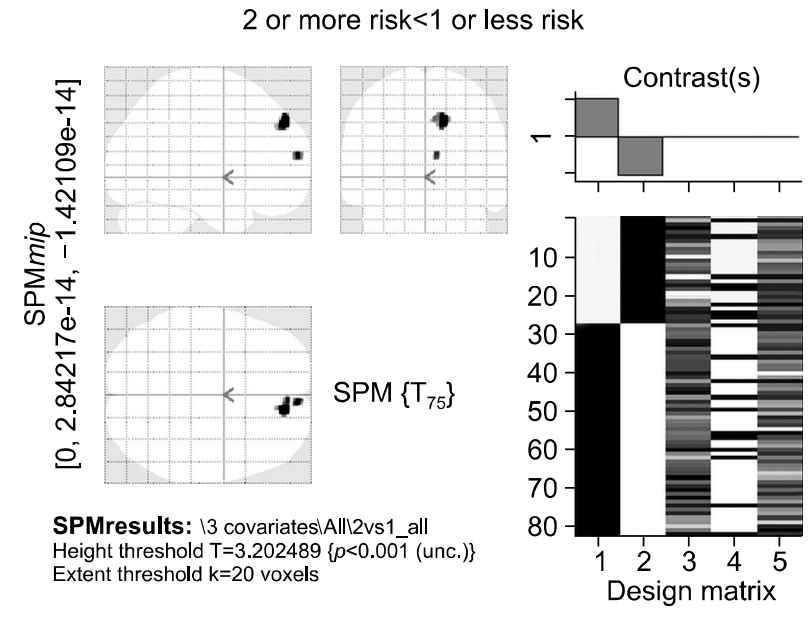

Fig. 5. Statistical parametric t-map showing reduced gray matter volumes linked to the additive effect of the three risk alleles $S$ (5HTTLPR), $G$ (rs4680) and T (rs6314) (subjects with 2 or 3 risk alleles $(n=53)$ vs. those with 1 or no risk alleles $(n=27)$ ), irrespective of phenotype, at an uncorrected significance threshold of $p<0.001$ and an extent threshold of 20 voxels. Total brain volume, age, and gender were entered in the two sample random effects analysis as co-variates. Display according to neurological convention (image left is participant's left).

5HTLPR, 5-hydroxy tryptamine transporter linked polymorphic region; SPM, statistical parametric mapping.

Table 9. Brain regions showing reduced gray matter volumes linked to the additive effect of the three risk alleles $S$ (5HTTLPR), $G(r s 4680)$ and $T$ (rs6314) irrespective of phenotype

\begin{tabular}{rrrrr}
\hline$X$ coor & $Y$ coor & $Z$ coor & Brain region & $Z$ score \\
\hline 11.13 & 39.49 & 43.34 & Right superior frontal gyrus* & 3.582761 \\
5.88 & 51.39 & 22.76 & Right medial frontal gyrus & 3.385971 \\
\hline
\end{tabular}

At an uncorrected significance threshold of $\mathrm{p}<0.001$ and an extent threshold of 20 voxels. Total brain volume, age, and gender were entered in the two sample random effects analysis as co-variates. *2 clusters significant at $p<0.001$ uncorrected; cluster with higher z-score listed.

carriers of the COMT rs4680 polymorphism and short allele carriers of the 5HTTLPR $44 \mathrm{bp}$ insertion/deletion polymorphism showed a trend $(p<0.001$, uncorrected) towards decreased regional brain volumes. Moreover, a trend (at $p<0.001$, uncorrected) towards additive effects of the above 3 risk alleles on brain morphology was also noted.

Whole brain VBM comparisons between phenotypes (schizophrenia vs. HCS) did not show definitive volumetric differences at a significance threshold of FWE $p$ $<0.05$. Circumscribed volumetric reductions in the left cerebellum were seen in patients with schizophrenia at FWEc threshold (cluster defining threshold: $p_{\text {uncorrected }}$ $<0.001 ; \mathrm{k}=793$ voxels) (Fig. 1A, Table 3), while more diffuse volumetric reductions were noted at an uncorrected threshold ( $p<0.001,20$ voxel extent threshold) (Fig. 1B, Table 4). A large body of evidence supports regional volumetric reductions in schizophrenia., 34,55 ) However the reported volumetric abnormalities vary from study to study, perhaps due to the effect of various confounding factors apart from differences in the constitution of genotypic risk variants, ${ }^{56)}$ such as age, gender distribution, age of onset, duration of illness, exposure to neuroleptics and drug use across the samples studied. ${ }^{4-8)}$ The absence of volumetric reductions in our sample of patients with schizophrenia at a stringent significance threshold (FWE $p<0.05$ ) may be due to the fact that the sample of patients with schizophrenia in the present study were predominantly comprised of paranoid and undifferentiated schizophrenia (none with residual schizophrenia), were relatively younger (mean age $=27.34$ years), had recent onset of illness (mean duration 24.54 months), with more than half of the subjects being neuroleptic-naïve/free (Table 1). Neuroleptic exposure and duration of illness have been shown to affect brain volumes. ${ }^{7,57)}$ Sub-group analysis of brain volumes between medicated $(n=16)$ and unmedicated $(n=25)$ patients with schizophrenia revealed significantly greater gray matter volumes in right anterior culmen of medicated patients at a statistical significance threshold of FWE $p$ $<0.05$ (Supplementary Fig. 4, Supplementary Table 4), which is in line with previous studies supporting increased gray matter volumes in patients with schizophrenia treated with anti-psychotics. ${ }^{7,57)}$ Since we only recruited patients with recent-onset (duration $<5$ years) illness, the variance on duration of illness of our sample was quite low and therefore, the effect of duration of illness on brain volumes could not be examined as above.

Carriers of the $\mathrm{T}$ allele (His452Tyr) of rs6314 polymorphism of 5HT2A were shown to have increased regional brain volumes in left inferior temporal and left inferior occipital gyri at a statistical significance threshold of FWEc $p<0.05$. The only previous study that has examined the association between the above polymorphism and brain morphology ${ }^{33)}$ reported volumetric reductions in the medial and lateral temporal brain regions in healthy subjects; however, the results of the above study were uncorrected for multiple comparisons $(p<0.001$, uncorrected). The possible pathophysiological significance of increased regional brain volumes in the left inferior temporal and left inferior occipital gyri and its relationship with the His452Tyr allele of rs6314 polymorphism of 5HT2A deserves more in-depth study, in the light of our 
preliminary findings.

Carriers of the Val allele of COMT rs4680 polymorphism showed decreased gray matter volumes in multiple brain regions spanning the frontal, temporal, parietal and occipital lobes as well as the cerebellum (Fig. 3, Table 6 ) in comparison to the Met homozygotes ( $p<0.001$, uncorrected). The Val/Val genotype of COMT is associated with higher enzymatic activity and increased catabolism of dopamine. ${ }^{17)}$ Val allele carriers were shown to have reduced volumes (at uncorrected statistical significance thresholds) of bilateral anterior cingulate ${ }^{51)}$ as well as temporal and frontal brain regions ${ }^{26,58)}$ similar to the results of our study. Thus the findings of the present study provide further support to previous reports of regional brain volumetric reductions associated with the Val allele of COMT rs4680 polymorphism.

Carriers of S allele of the 5HTTLPR polymorphism showed decreased gray matter volumes in the right anterior culmen and middle frontal gyrus when compared to the L homozygotes ( $p<0.001$, uncorrected). A previous study in HCS has reported reduced brain volumes in perigenual anterior cingulate cortex and amygdala ${ }^{40)}$ in carriers of the short allele of the 5HTTLPR polymorphism. However, in a post-mortem study carried out in subjects who were diagnosed to have schizophrenia, the $\mathrm{S}$ allele was reported to be associated with a higher number and volume of pulvinar neurons in the thalamus. ${ }^{41)}$ Therefore, further studies in larger samples of healthy subjects and patients with schizophrenia are required to establish the effect of S allele of the 5HTTLPR rs25531 polymorphism on brain volumes.

Exploration of the additive effect of the three risk alleles, viz., S (5HTTLPR), G (COMT rs4680) and T (5HT2A rs6314) revealed trend-level $(p<0.001)$ reductions in brain volumes in the right superior frontal and medial frontal gyri in the high risk group (containing 2 or 3 risk alleles) when compared to the low risk group (containing 1 or no risk alleles). It is to be noted that the high risk group $(n=53)$ contained only 13 subjects with the $\mathrm{T}$ allele of the rs6314 polymorphism of 5HT2A that predisposes to greater regional brain volumes. Thus the high risk group $(\mathrm{N}=53)$ had a preponderance of subjects with the $\mathrm{G}(\mathrm{GG}[\mathrm{n}=21]$; $\mathrm{AG}[\mathrm{n}=30])$ and $\mathrm{S}(\mathrm{SS}[\mathrm{n}=22]$; LS $[\mathrm{n}=29])$ risk alleles of COMT (rs4680) and 5HTT (5HTTLPR) respectively. The above alleles were shown to be associated with lower regional brain volumes (see above), which may be the reason why the high risk group showed an overall trend toward volumetric reductions. When the exploration of additive effects was restricted to only those having the T allele of 5HT2A (rs6314) polymorphism $(n=13)$ along with one or two of the other two risk alleles, the high risk group showed increased regional gray matter volumes. This clearly shows that the presence of $\mathrm{T}$ allele of rs6314 polymorphism of 5HT2A is contributing to greater gray matter volumes in the brain. Exploration of the additive effects of the risk alleles of the COMT rs4680 and 5HTTLPR polymorphisms, excluding the subjects with the T allele of the 5HT2A rs6314 polymorphism, revealed regional gray matter volumetric reductions (Supplementary Table 3, Supplementary Fig. 3). Therefore the results of the additive analysis including all the three risk alleles demonstrates how regional brain volumes are not only a function of the effect of a given risk allele on regional brain volumes but also on the frequency of the risk allele in a given sample as well as the cumulative effect of the frequency distribution of various other risk alleles that may impact regional brain volumes. This cumulative effect of various risk alleles on regional brain volumes in a given sample could possibly explain the inconsistency of brain morphometric findings across various samples of patients with schizophrenia. However, the limited size of the sample of individuals with the risk allele $\mathrm{T}$ $(n=14)$ of 5HT2A (rs6314) polymorphism should be taken into consideration while interpreting the results of the study.

The results of the present study demonstrate how brain volumes are influenced not only by the phenotype (schizophrenia vs. healthy), but also by the genotypic risk variants of schizophrenia risk genes affecting monoamine signaling. When brain volumes are compared across phenotypes within the overall sample, genotypic differences are overlooked, while in the present study, instead of phenotypic differences, we examined volumetric differences across genotypes. There were differences in the extent (5HT2A His452Tyr $>$ COMTVal158Met $>$ 5HTTLPR 44 bp insertion/deletion) and direction (5HT2A His452Tyr: volumetric increase; COMT Val158Met and 5HTTLPR $44 \mathrm{bp}$ insertion/deletion: volumetric decreases) of volumetric changes associated with the genotypic risk variants of the above three risk genes. Such differences in the influence of polymorphisms of the various schizophrenia risk genes on brain volumes along with the variability in other confounding factors discussed before might explain the variability of morphometric findings (volumetric increases, ${ }^{59)}$ decreases $^{33)}$ or no difference ${ }^{60)}$ ) across multiple studies.

This study explored the effect on brain morphometry of polymorphisms of three schizophrenia risk genes that me- 
diate monoamine signaling in a sample of 80 subjects comprising of 41 patients with schizophrenia and 39 HCS. There are no previous reports of the effect of the above 3 gene polymorphisms individually as well as additively on brain morphometry in the same study. Though the overall sample size was not too small, the number of individuals in the genotype-wise sub-groups was limited. Thus, given the modest sample sizes, the findings of this exploratory analysis need further confirmation in larger samples.

In conclusion, we have demonstrated higher regional brain volumes associated with the $\mathrm{T}$ allele of the rs 6314 polymorphism of 5HT2A in the left inferior temporal and inferior occipital gyri. A trend towards lower regional brain volumes associated with the Val allele of the COMT rs4680 polymorphism as well as the short allele of the 5HTTLPR 44 bp insertion/deletion polymorphism was noted. We have also demonstrated a trend towards additive effects of the above 3 risk alleles on brain morphology. This could mean that variability in the genetic profiles of the study samples could be one of the reasons for inconsistent findings of previous morphometric studies in schizophrenia and other neuropsychiatric conditions. Perhaps the reported volumetric alterations are an independent function of the individual and additive effects of the multiple schizophrenia risk genes on brain volume, rather than a function of the disorder per se. This possibility needs to be explored in larger samples of patients with schizophrenia using in silico methods or alternately using complex genetically engineered animal models.

\section{- Acknowledgments}

The authors acknowledge funding support from the Department of Biotechnology (DBT), Government of India (Grant No. BT/PR/8363/MED/14/1252 to J.P.J.).

The authors would like to acknowledge Dr Bhavani Shankara Bagepally, Dr Nagaraj Moily, Ms Ammu Lukose, and Dr Vikram Arunachalam, research Fellows at MBIAL and Molecular Genetics Laboratory at NIMHANS for their kind assistance in subject recruitment and data compilation.

\section{REFERENCES}

1. Wright IC, Rabe-Hesketh S, Woodruff PW, David AS, Murray RM, Bullmore ET. Meta-analysis of regional brain volumes in schizophrenia. Am J Psychiatry 2000;157:16-25.

2. Steen RG, Mull C, McClure R, Hamer RM, Lieberman JA. Brain volume in first-episode schizophrenia: systematic review and meta-analysis of magnetic resonance imaging studies. Br J Psychiatry 2006;188:510-518.

3. Shenton ME, Dickey CC, Frumin M, McCarley RW. A review of MRI findings in schizophrenia. Schizophr Res
2001;49:1-52.

4. Burke L, Androutsos C, Jogia J, Byrne P, Frangou S. The Maudsley Early Onset Schizophrenia Study: the effect of age of onset and illness duration on fronto-parietal gray matter. Eur Psychiatry 2008;23:233-236.

5. Barnes J, Ridgway GR, Bartlett J, Henley SM, Lehmann M, Hobbs $\mathrm{N}$, et al. Head size, age and gender adjustment in MRI studies: a necessary nuisance? Neuroimage 2010;53: 1244-1255.

6. Schwarzkopf SB, Olson SC, Coffman JA, Nasrallah HA. Third and lateral ventricular volumes in schizophrenia: support for progressive enlargement of both structures. Psychopharmacol Bull 1990;26:385-391.

7. Keshavan MS, Bagwell WW, Haas GL, Sweeney JA, Schooler NR, Pettegrew JW. Changes in caudate volume with neuroleptic treatment. Lancet 1994;344:1434.

8. Borne J, Riascos R, Cuellar H, Vargas D, Rojas R. Neuroimaging in drug and substance abuse part II: opioids and solvents. Top Magn Reson Imaging 2005;16:239-245.

9. Bennett CM, Wolford GL, Miller MB. The principled control of false positives in neuroimaging. Soc Cogn Affect Neurosci 2009;4:417-422.

10. Ioannidis JP. Excess significance bias in the literature on brain volume abnormalities. Arch Gen Psychiatry 2011;68:773-780.

11. Harrison PJ, Weinberger DR. Schizophrenia genes, gene expression, and neuropathology: on the matter of their convergence. Mol Psychiatry 2005;10:40-68.

12. Girard SL, Dion PA, Rouleau GA. Schizophrenia genetics: putting all the pieces together. Curr Neurol Neurosci Rep 2012;12:261-266.

13. McGuffin P, Owen MJ, Farmer AE. Genetic basis of schizophrenia. Lancet 1995;346:678-682.

14. Camarena B, Fresán A, Aguilar A, Escamilla R, Saracco R, Palacios $\mathrm{J}$, et al. Monoamine oxidase $a$ and $B$ gene polymorphisms and negative and positive symptoms in schizophrenia. ISRN Psychiatry 2012;2012:852949.

15. Wei YL, Li CX, Li SB, Liu Y, Hu L. Association study of monoamine oxidase $A / B$ genes and schizophrenia in Han Chinese. Behav Brain Funct 2011;7:42.

16. Ehrlich S, Morrow EM, Roffman JL, Wallace SR, Naylor M, Bockholt HJ, et al. The COMT Val108/158Met polymorphism and medial temporal lobe volumetry in patients with schizophrenia and healthy adults. Neuroimage 2010;53:9921000.

17. Lachman HM, Papolos DF, Saito T, Yu YM, Szumlanski CL, Weinshilboum RM. Human catechol-O-methyltransferase pharmacogenetics: description of a functional polymorphism and its potential application to neuropsychiatric disorders. Pharmacogenetics 1996;6:243-250.

18. Shifman S, Bronstein M, Sternfeld M, Pisanté-Shalom A, Lev-Lehman E, Weizman A, et al. A highly significant association between a COMT haplotype and schizophrenia. Am J Hum Genet 2002;71:1296-1302.

19. Glatt SJ, Faraone SV, Tsuang MT. Association between a functional catechol O-methyltransferase gene polymorphism and schizophrenia: meta-analysis of case-control and family-based studies. Am J Psychiatry 2003;160:469-476.

20. Voisey J, Swagell CD, Hughes IP, Lawford BR, Young RM, Morris CP. HapMap tag-SNP analysis confirms a role for COMT in schizophrenia risk and reveals a novel association. Eur Psychiatry 2012;27:372-376.

21. Hoenicka J, Garrido E, Martínez I, Ponce G, Aragüés M, Rodríguez-Jiménez R, et al; PARGPARG. Gender-specific COMT Val158Met polymorphism association in Spanish 
schizophrenic patients. Am J Med Genet B Neuropsychiatr Genet 2010;153B:79-85.

22. Gupta M, Bhatnagar P, Grover S, Kaur H, Baghel R, Bhasin $\mathrm{Y}$, et al. Association studies of catechol-O-methyltransferase (COMT) gene with schizophrenia and response to antipsychotic treatment. Pharmacogenomics 2009;10:385-397.

23. Sullivan PF, Lin D, Tzeng JY, van den Oord E, Perkins D, Stroup TS, et al. Genomewide association for schizophrenia in the CATIE study: results of stage 1. Mol Psychiatry 2008; 13:570-584.

24. Apud JA, Weinberger DR. Treatment of cognitive deficits associated with schizophrenia: potential role of catechol-Omethyltransferase inhibitors. CNS Drugs 2007;21:535-557.

25. Goldberg TE, Egan MF, Gscheidle T, Coppola R, Weickert $\mathrm{T}$, Kolachana BS, et al. Executive subprocesses in working memory: relationship to catechol-O-methyltransferase Val158Met genotype and schizophrenia. Arch Gen Psychiatry 2003;60:889-896.

26. Ohnishi T, Hashimoto R, Mori T, Nemoto K, Moriguchi Y, Iida $\mathrm{H}$, et al. The association between the Val158Met polymorphism of the catechol-O-methyl transferase gene and morphological abnormalities of the brain in chronic schizophrenia. Brain 2006;129:399-410.

27. Honea R, Verchinski BA, Pezawas L, Kolachana BS, Callicott $\mathrm{JH}$, Mattay VS, et al. Impact of interacting functional variants in COMT on regional gray matter volume in human brain. Neuroimage 2009;45:44-51.

28. Erdmann J, Shimron-Abarbanell D, Rietschel M, Albus M, Maier W, Körner J, et al. Systematic screening for mutations in the human serotonin-2A (5-HT2A) receptor gene: identification of two naturally occurring receptor variants and association analysis in schizophrenia. Hum Genet 1996;97:614-619.

29. Ni J, Lu W, Wu Z, Chen J, Yi Z, Zhang C. T102C polymorphism of serotonin $2 A$ type receptor gene confers susceptibility to (early onset) schizophrenia in Han Chinese: an association study and meta-analysis. Asia Pac Psychiatry 2013;5:24-30.

30. Tay AH, Lim LC, Lee WL, Wong KE, Wong LY, Tsoi WF. Association between allele 1 of T102C polymorphism, 5-hydroxytryptamine 2 a receptor gene and schizophrenia in Chinese males in Singapore. Hum Hered 1997;47:298-300.

31. Abdolmaleky HM, Faraone SV, Glatt SJ, Tsuang MT. Meta-analysis of association between the T102C polymorphism of the 5HT2a receptor gene and schizophrenia. Schizophr Res 2004;67:53-62.

32. Williams J, Spurlock G, McGuffin P, Mallet J, Nöthen MM, Gill M, et al. Association between schizophrenia and T102C polymorphism of the 5-hydroxytryptamine type 2a-receptor gene. European Multicentre Association Study of Schizophrenia (EMASS) Group. Lancet 1996;347:1294-1296.

33. Filippini N, Scassellati C, Boccardi M, Pievani M, Testa C, Bocchio-Chiavetto L, et al. Influence of serotonin receptor $2 A$ His452Tyr polymorphism on brain temporal structures: a volumetric MR study. Eur J Hum Genet 2006;14:443-449.

34. Lesch KP, Mössner R. Genetically driven variation in serotonin uptake: is there a link to affective spectrum, neurodevelopmental, and neurodegenerative disorders? Biol Psychiatry 1998;44:179-192.

35. Pae CU, Kim JJ, Lee SJ, Lee CU, Lee C, Paik IH, et al. Polymorphism of the serotonin transporter gene and symptomatic dimensions of schizophrenia in the Korean population. Neuropsychobiology 2003;47:182-186.

36. Golimbet VE, Alfimova MV, Shcherbatikh T, Kaleda VG, Abramova LI, Rogaev EI. Serotonin transporter gene polymorphism and schizoid personality traits in the patients with psychosis and psychiatrically well subjects. World $J$ Biol Psychiatry 2003;4:25-29.

37. Bayle FJ, Leroy S, Gourion D, Millet B, Olié JP, Poirier $\mathrm{MF}$, et al. 5HTTLPR polymorphism in schizophrenic patients: further support for association with violent suicide attempts. Am J Med Genet B Neuropsychiatr Genet 2003;119B:13-17.

38. Park SH, Kim MC, Yoon SH, Kong BG, Kim YH. Association between serotonin transporter polymorphism and the effect of atypical antipsychotics on serum glucose and lipids. Korean J Psychopharmacol 2004;15:196-205.

39. Vijayan NN, Iwayama Y, Koshy LV, Natarajan C, Nair C, Allencherry PM, et al. Evidence of association of serotonin transporter gene polymorphisms with schizophrenia in a South Indian population. J Hum Genet 2009;54:538-542.

40. Pezawas L, Meyer-Lindenberg A, Drabant EM, Verchinski BA, Munoz KE, Kolachana BS, et al. 5-HTTLPR polymorphism impacts human cingulate-amygdala interactions: a genetic susceptibility mechanism for depression. Nat Neurosci 2005;8:828-834.

41. Young KA, Holcomb LA, Bonkale WL, Hicks PB, Yazdani $\mathrm{U}$, German DC. 5HTTLPR polymorphism and enlargement of the pulvinar: unlocking the backdoor to the limbic system. Biol Psychiatry 2007;61:813-818.

42. McIntosh AM, Baig BJ, Hall J, Job D, Whalley HC, Lymer GK, et al. Relationship of catechol-O-methyltransferase variants to brain structure and function in a population at high risk of psychosis. Biol Psychiatry 2007;61:1127-1134.

43. Kay SR, Fiszbein A, Opler LA. The positive and negative syndrome scale (PANSS) for schizophrenia. Schizophr Bull 1987;13:261-276.

44. Woods SW. Chlorpromazine equivalent doses for the newer atypical antipsychotics. J Clin Psychiatry 2003;64:663-667.

45. Taylor D, Paton C, Kerwin S. The South London and Maudsley NHS Foundation Trust and Oxleas NHS Foundation Trust Prescribing Guidelines. 10th ed. London: Informa Healthcare; 2009.

46. Kroken RA, Johnsen E, Ruud T, Wentzel-Larsen T, Jørgensen HA. Treatment of schizophrenia with antipsychotics in Norwegian emergency wards, a cross-sectional national study. BMC Psychiatry 2009;9:24.

47. Ashburner J, Friston KJ. Unified segmentation. Neuroimage 2005;26:839-851.

48. Miller SA, Dykes DD, Polesky HF. A simple salting out procedure for extracting DNA from human nucleated cells. Nucleic Acids Res 1988;16:1215.

49. Ruiz-Sanz JI, Aurrekoetxea I, Ruiz del Agua A, Ruiz-Larrea MB. Detection of catechol-O-methyltransferase Val158Met polymorphism by a simple one-step tetra-primer amplification refractory mutation system-PCR. Mol Cell Probes 2007;21:202-207.

50. Wendland JR, Martin BJ, Kruse MR, Lesch KP, Murphy DL. Simultaneous genotyping of four functional loci of human SLC6A4, with a reappraisal of 5-HTTLPR and rs25531. Mol Psychiatry 2006;11:224-226.

51. Zinkstok J, Schmitz N, van Amelsvoort T, de Win M, van den Brink W, Baas F, et al. The COMT val158met polymorphism and brain morphometry in healthy young adults. Neurosci Lett 2006;405:34-39.

52. Hranilovic D, Stefulj J, Schwab S, Borrmann-Hassenbach M, Albus M, Jernej B, et al. Serotonin transporter promoter and intron 2 polymorphisms: relationship between allelic variants and gene expression. Biol Psychiatry 2004;55: 1090-1094. 
53. McNab F, Leroux G, Strand F, Thorell L, Bergman S, Klingberg T. Common and unique components of inhibition and working memory: an fMRI, within-subjects investigation. Neuropsychologia 2008;46:2668-2682.

54. Shenton ME, Whitford TJ, Kubicki M. Structural neuroimaging in schizophrenia: from methods to insights to treatments. Dialogues Clin Neurosci 2010;12:317-332.

55. Honea R, Crow TJ, Passingham D, Mackay CE. Regional deficits in brain volume in schizophrenia: a meta-analysis of voxel-based morphometry studies. Am J Psychiatry 2005; 162:2233-2245.

56. Zhang JP, Malhotra AK. Genetics of schizophrenia: What do we know? Curr Psychiatr 2013;12:24-33.

57. Deng MY, McAlonan GM, Cheung C, Chiu CP, Law CW, Cheung $\mathrm{V}$, et al. A naturalistic study of grey matter volume increase after early treatment in anti-psychotic naïve, newly diagnosed schizophrenia. Psychopharmacology (Berl) 2009;206:437-446.

58. Taylor WD, Züchner S, Payne ME, Messer DF, Doty TJ, MacFall JR, et al. The COMT Val158Met polymorphism and temporal lobe morphometry in healthy adults. Psychiatry Res 2007; 155:173-177.

59. Potvin S, Mancini-Marïe A, Fahim C, Mensour B, Lévesque $\mathrm{J}$, Karama S, et al. Increased striatal gray matter densities in patients with schizophrenia and substance use disorder: a voxel-based morphometry study. Psychiatry Res 2007; 154:275-279.

60. Dutt A, McDonald C, Dempster E, Prata D, Shaikh M, Williams I, et al. The effect of COMT, BDNF, 5-HTT, NRG1 and DTNBP1 genes on hippocampal and lateral ventricular volume in psychosis. Psychol Med 2009;39:1783-1797. 
Interaction: genotype $\mathrm{x}$ diagnosis

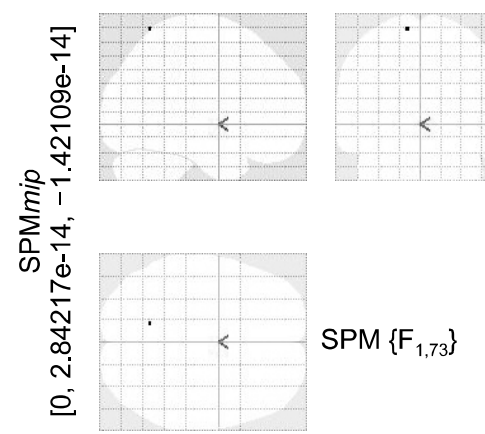

SPMresults: DINew_sz_llpaperlpriya_listyr Height threshold $\mathrm{F}=26.005090\{p<0.05$ (FWE) $\}$ Height threshold $\mathrm{F}=26.005090$
Extent threshold $\mathrm{k}=0$ voxels

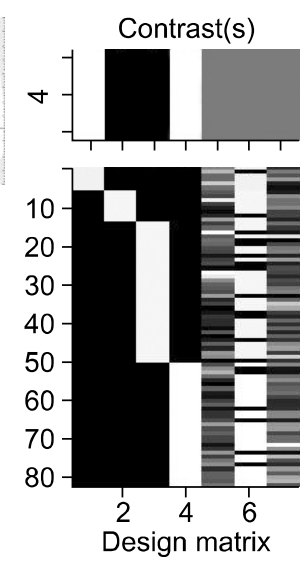

Supplementary Fig. 1. Statistical parametric t-map showing $5 \mathrm{HT} 2 \mathrm{~A} \times$ diagnosis interaction effects on brain morphology at family wise error $(p<0.05)$. Display according to neurological convention (image left is participant's left).

5-HT2A, 5-hydroxy tryptamine 2A; SPM, statistical parametric mapping.

Supplementary Table 1. Brain regions showing $5-\mathrm{HT} 2 \mathrm{~A} \times$ diagnosis interaction effects on brain morphology

\begin{tabular}{ccccc}
\hline$X$ coor & $Y$ coor & $Z$ coor & Brain region & $Z$ score \\
\hline-13.08 & -63.61 & 60.19 & Left superior Parietal Lobule & 4.724314 \\
\hline
\end{tabular}

Family wise error: $p<0.05$.
$\mathrm{TC}>1$ or less
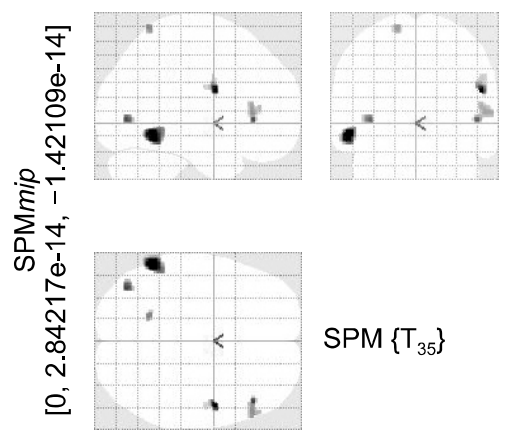

Contrast(s)

SPMresults: $\mid \mathrm{TCl} 1 \mathrm{~s}$ or less

Height threshold $\mathrm{T}=3.340045\{p<0.001$ (unc.) $\}$ Extent threshold $k=20$ voxels
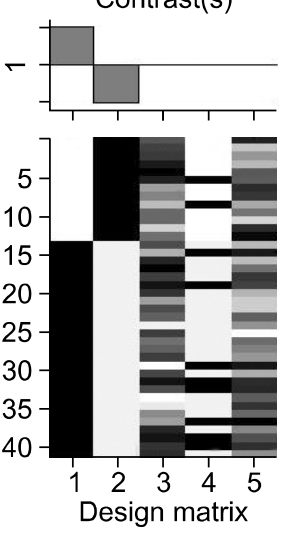

Supplementary Fig. 2. Statistical parametric t-map showing increased gray matter volumes in those subjects having the $T$ allele of 5HT2A (rs6314) polymorphism along with one or two of the other two risk alleles $(n=13)$ vs. those who had one or no risk alleles $(n=27)$ (uncorrected significance threshold of $p<0.001$ and an extent threshold of 20 voxels), irrespective of phenotype. Total brain volume, age, and gender were entered in the two sample random effects analysis as co-variates. Display according to neurological convention (image left is participant's left).

5HT2A, 5-hydroxy tryptamine 2A; TC, tyrosine-cysteine; CC, cysteinecysteine; SPM, statistical parametric mapping.

Supplementary Table 2. Brain regions showing increased gray matter volumes in those subjects having the T allele of $5 \mathrm{HT} 2 \mathrm{~A}$ (rs6314) polymorphism along with one or two of the other two risk alleles $(n=13)$ versus those who had one or no risk alleles $(n=27)$

\begin{tabular}{rrrll}
\hline$X$ coor & $Y$ coor & $Z$ coor & Brain region & $Z$ score \\
\hline 45.91 & -5.37 & 24.81 & Right precentral gyrus* & 3.904783 \\
-52.39 & -50.89 & -10.89 & Left inferior temporal gyrus & 3.893673 \\
-37.31 & -71.58 & -1.78 & Left inferior occipital gyrus & 3.581914 \\
43.45 & 25.82 & 8.81 & Right inferior frontal gyrus* & 3.576762 \\
-17.24 & -59.4 & 60.52 & Left superior parietal lobule & 3.399359 \\
\hline
\end{tabular}

Uncorrected significance threshold of $p<0.001$ and an extent threshold of 20 voxels; irrespective of phenotype. Total brain volume, age, and gender were entered in the two sample random effects analysis as co-variates.

*2 clusters significant at $p<0.001$ uncorrected; cluster with higher z-score listed. 
2 risk $<1$ or less risk

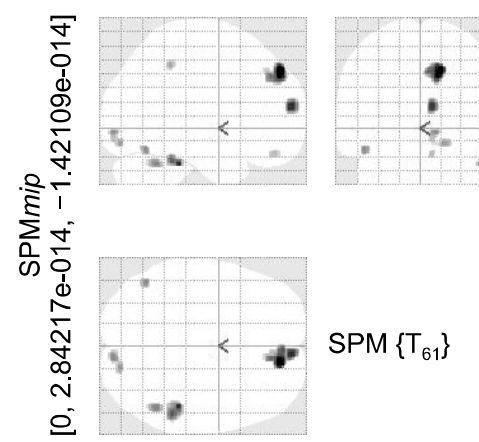

SPMresults: ICornt_Ipr_excluding TC Height threshold $T=3.229296\{p<0.001$ (unc.) $\}$ Extent threshold $\mathrm{k}=20$ voxels

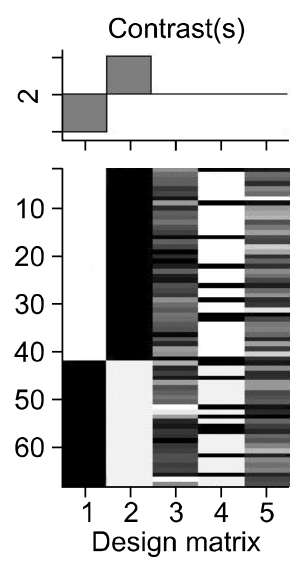

Supplementary Fig. 3. Statistical parametric t-map showing reduced gray matter volumes in subjects carrying risk alleles of Val allele of COMT and short allele of $5 H T T(n=40)$ vs. those subjects having 1 or no risk allele of the COMT and $5 \mathrm{HTT}$ polymorphisms $(n=26)$ (uncorrected significance threshold of $p<0.001$ and an extent threshold of 20 voxels), irrespective of phenotype. Total brain volume, age, and gender were entered in the two sample random effects analysis as co-variates. Display according to neurological convention (image left is participant's left).

Val, valine; COMT, catechol o-methyltransferase; 5HTT, 5-hydroxy tryptamine transporter; SPM, statistical parametric mapping.

Supplementary Table 3. Brain regions showing reduced gray matter volumes in subjects carrying risk alleles of Val allele of COMT and short allele of $5 \mathrm{HTT}(\mathrm{n}=40)$ and those subjects having 1 or no risk allele of the COMT and 5HT polymorphisms $(n=26)$

\begin{tabular}{rrrll}
\hline$X$ coor & $Y$ coor & $Z$ coor & Brain region & $Z$ score \\
\hline 11.11 & 39.36 & 44.68 & Right superior frontal gyrus* & 4.099232 \\
8.37 & 32.78 & 39.95 & Right medial frontal gyrus* & 3.610037 \\
43.61 & -49.82 & -25.38 & Right anterior culmen & 3.503955 \\
8.53 & -85.27 & -7.71 & Right lingual gyrus & 3.487104 \\
-43.99 & -60.06 & -18.37 & Left posterior declive & 3.477983 \\
15.59 & -80.33 & -15.23 & Right posterior declive & 3.409085 \\
46.93 & -46.46 & 41.21 & Right inferior parietal lobule & 3.365151 \\
6.27 & 40.55 & -10.69 & Right anterior cingulate & 3.278009 \\
\hline
\end{tabular}

Uncorrected significance threshold of $p<0.001$ and an extent threshold of 20 voxels: irrespective of phenotype. Total brain volume, age, and gender were entered in the two sample random effects analysis as co-variates.

*2 clusters significant at $p<0.001$ uncorrected; cluster with higher z-score listed.

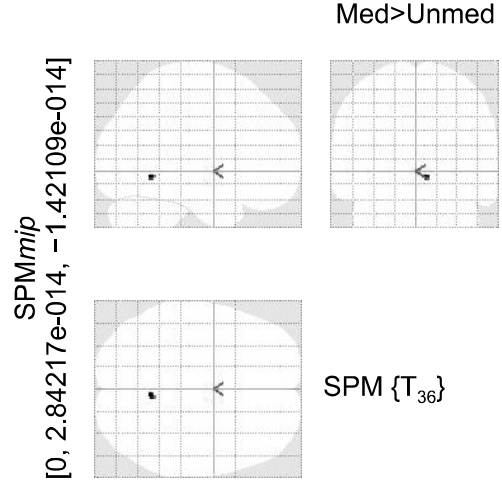

SPMresults: |review analysis\med_umed Height threshold T=5.358919 $\{p<0.05$ (FWE) Height threshold $\mathrm{T}=5.358919$
Extent threshold $\mathrm{k}=0$ voxels

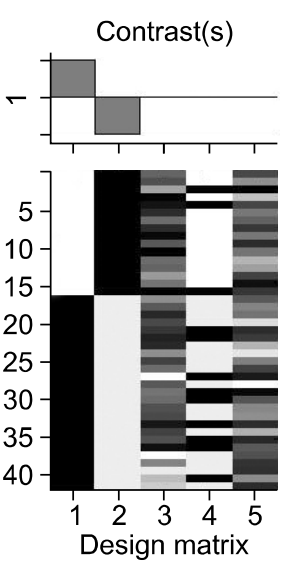

Supplementary Fig. 4. Statistical parametric t-map showing greater gray matter volumes in medicated schizophrenia subjects (Med, $\mathrm{n}=16$ ) in comparison to unmedicated patients with schizophrenia (Unmed, $\mathrm{n}=25$ ) at a significance threshold of $p<0.05$; family wise error corrected when total brain volume, age, and gender were entered in the two sample random effects analysis as co-variates. Display according to neurological convention (image left is participant's left).

SPM, statistical parametric mapping.

Supplementary Table 4 . Brain regions showing greater gray matter volumes in medicated schizophrenia subjects $(n=16)$ in comparison to unmedicated patients with schizophrenia $(n=25)$

\begin{tabular}{ccccc}
\hline$X$ coor & $Y$ coor & $Z$ coor & Brain region & Z score \\
\hline 4.47 & -52.98 & -6.07 & Right anterior culmen & 4.7256 \\
\hline
\end{tabular}

At a significance threshold of $p<0.05$; family wise error corrected when total brain volume, age, and gender were entered in the two sample random effects analysis as co-variates. 